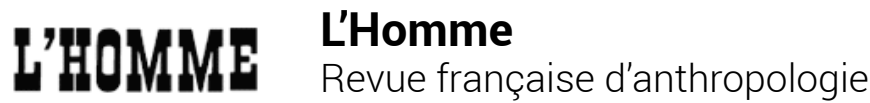

195-196 | 2010

Auto-biographie, Ethno-biographie

\section{Quelques jours de la vie d'Homère}

A few Days in the Life of Homer

\section{Dominique Casajus}

\section{(2) OpenEdition}

\section{Journals}

Édition électronique

URL : http://journals.openedition.org/lhomme/22538

DOI : 10.4000/lhomme.22538

ISSN : 1953-8103

\section{Éditeur}

Éditions de l'EHESS

\section{Édition imprimée}

Date de publication : 10 novembre 2010

Pagination : 333-358

ISSN : 0439-4216

Référence électronique

Dominique Casajus, «Quelques jours de la vie d'Homère », L'Homme [En ligne], 195-196 | 2010, mis en ligne le 04 novembre 2012, consulté le 01 mai 2019. URL : http://journals.openedition.org/ Ihomme/22538; DOl : 10.4000/lhomme.22538 


\title{
Quelques jours de la vie d'Homère
}

\author{
Dominique Casajus
}

Q

UAND, ENTRE 1666 et 1670, François Hédelin, abbé d'Aubignac, entreprit d'écrire ses Conjectures académiques ou dissertation sur l'Iliade, il se doutait bien que son propos risquait d'être tenu pour blasphématoire. Aussi ne l'ouvrit-il qu'avec d'infinies précautions :

"Le sujet de ce discours est de ceux que de plus savants que moi pourroient condamner, mais il n'en faut rien craindre de funeste: on peut soûtenir qu'Homere n'étoit pas un bon poëte, \& que même il n'a jamais été, sans se rendre suspect d'être mal affectionné à la couronne, ni de mal penser de la religion ; l'on ne sera pas exposé pour cela aux peines des loix, ni aux anathêmes de l'Église ; \& cette opinion, singuliere à la vérité, n’obligera personne à se défendre des orages de la Cour, ni des foudres du Vatican $^{1} »(1715: 5-6)$.

Il n'affronta ni l'orage ni la foudre; mais l'académicien François Charpentier, qu'on avait chargé d'examiner le manuscrit, lui représenta que "cet ouvrage, quoique rempli d'érudition, ne lui ferait pas d'honneur ${ }^{2}$ ». L'abbé insista, Charpentier ne céda pas, et le livre ne fut publié qu'en 1715, quarante ans après la mort de l'auteur et treize ans après celle du censeur. En fait, le manuscrit avait déjà beaucoup circulé, et, dans la querelle qui avait fait rage entre-temps, le parti des Modernes ne s'était pas privé d'y puiser des munitions. L'opinion qu'Homère "n'étoit pas un bon poëte "était donc devenue banale. Affirmer qu'il n’avait "jamais été " l'était beaucoup moins. Charles Perrault lui-même avait reculé devant tant d'iconoclasme. Le dialogue à la manière de Platon qu'il publia entre 1688 et 1697 faisait de longues et louangeuses allusions à l'abbé d'Aubignac,

1. Pour la date de la composition de l'ouvrage, voir Magnien (1925: XII sq.)

2. Le propos est rapporté par Magnien (ibid. : XVIII-XXIV). 
mais le personnage qui y portait la parole de l'auteur se contentait de remarquer que "beaucoup d'excellens Critiques soûtiennent qu'il n'y a jamais eu au monde un homme nommé Homère, qui ait composé les vingt quatre livres de l'Iliade, \& les vingt quatre livres de l'Odyssée " (Perrault 1692: 32-33). Bien entendu, comme Boileau le releva ironiquement, ces excellents critiques "se réduis[ai]ent à feu M. l'abbé d'Aubignac" (1837: 351); mais, dans une matière aussi sulfureuse, mieux valait clamer qu'on avait le nombre pour soi. Le champion des modernes était prudent; et surtout, il avait été un peu effarouché. Effarouchés eux aussi, les éditeurs des Conjectures préférèrent avertir le lecteur que les hypothèses de l'auteur pourraient paraître "singulieres " ${ }^{3}$.

Il faut dire que les «vies" d'Homère qui circulaient depuis l'Antiquité avaient de quoi rendre perplexe. Elles ne s'accordaient ni sur l'identité de ses parents, ni sur les lieux et l'époque de sa naissance et de sa vie, ni sur les ouvrages à lui attribuer, et il y avait même des doutes sur son véritable nom. Et l'abbé d'observer non sans bon sens qu' «il est impossible qu'un homme ait vêcu parmi les autres sans nom, qu'il soit né sans pere ni mere, qu'il ait vêcu sur la terre sans naître en quelque lieu, qu'il ait passé un nombre d'années assez considerable, sans qu'il se trouve dans la suite des temps, qu'on ne sçache point le temps de sa mort, \& que ses ouvrages aient été si mal connus de tous les plus anciens auteurs " (Aubignac 1715: 82). L'affaire était donc entendue: Homère n'avait jamais existé, les ouvrages qu'on lui attribuait n'étaient "qu'un recüeil de chansons cousuës, un amas de plusieurs pièces auparavant dispersées, \& depuis jointes ensemble " (Ibid. : 82-83). Pour ce qui est du nom "Homère " lui-même, l'origine s'en expliquait aisément: "le Compilateur qui n'y mettoit rien du sien, ne voulant pas l'intituler de son nom, s'avisa de le nommer le rapsodie d'Homere, c'est-à-dire le recüeil des chansons de l'Aveugle, parce que ces pieces étoient communément chantées depuis long-temps par des aveugles aux portes des Bourgeois " (Ibid.: 93-94). Bien que l'abbé se réclamât de Flavius Josèphe (Ibid. : 100), il était beaucoup plus expéditif que lui. L'auteur du Contre Appion disait seulement qu'Homère n'avait pas laissé d'écrits, il ne le faisait pas disparaître de l'Histoire. C'était maintenant chose faite, de sorte qu'il n'y avait plus lieu de s'interroger ni sur l'identité ni sur la vie d'Homère.

Dix ans après la parution des Conjectures académiques, Giambattista Vico publia la première édition de sa Scienza nuova, où il retraçait majestueusement l'histoire des décrets par lesquels la Providence «a gouverné la grande cité du genre humain" (Michelet 1827 : XIII). Une seconde

3. Cf. Aubignac (1715), à la première page, non numérotée, de l'Avis au lecteur. 
édition suivrait en 1730 , puis une troisième en 1744 . Lui aussi avait des doutes sur l'existence d'Homère, mais, du moins à partir de la deuxième édition, il ne les exprimait pas du tout comme l'abbé d'Aubignac ${ }^{4}$. On lit en effet au troisième livre de l'ouvrage, intitulé «Découverte du véritable Homère ${ }^{5}$ : "Homère a été l'idéal ou le caractère héroïque $d u$ peuple de la Grèce racontant sa propre histoire dans des chants nationaux". Si on était incertain sur la patrie d'Homère, «si les peuples de la Grèce se disputèrent l'honneur de lui avoir donné le jour [...], c'est qu'ils étaient eux-mêmes Homère"; s'il y avait une telle diversité d'opinion sur l'époque où il avait vécu, "c'est qu'il vécut en effet dans la bouche et dans la mémoire des mêmes peuples, depuis la guerre de Troie jusqu'au temps de Numa, ce qui fait quatre cent soixante ans " (Vico 1827 [1744] : 278-279). Il était donc vain de se mettre en quête d'un personnage historique baptisé " Homère ", mais il y avait bien une réalité derrière ce nom; l'appellation qu'elle avait reçue venait de ce que les rhapsodes qui allaient dans les marchés en chantant l'histoire des peuples grecs étaient généralement aveugles, infirmité supposée aller de pair avec une mémoire étonnante (Ibid. : 277). Et, de la mémoire, il leur en fallait puisque cela se passait à une époque où l'écriture était encore inconnue. Nous ne sommes pas très loin des Conjectures académiques, mais le ton n'est vraiment pas le même. L'abbé d'Aubignac avait réduit à néant le personnage d'Homère, et s'en était fort amusé ; Vico l'avait dilaté aux dimensions de la Grèce, et même de l'humanité tout entière en son âge héroïque.

L'abbé était un bel esprit, Vico un jurisconsulte et un philosophe. Rousseau, qui eût exécré le premier et se fût sans doute trouvé quelque affinité avec le second, les rejoignait tous deux au moins sur un point. Intitulé "S'il est probable qu'Homère ait su écrire », le chapitre VI de son Essai sur l'origine des langues concluait un développement vraisemblablement inspiré de Flavius Josèphe par cette maxime : «Les autres Poetes écrivoient, Homere seul avait chanté ${ }^{6}(O . C ., V: 390)$. Homère donc n'avait pas été un homme de plume, mais Rousseau ne doutait pas qu'il eût existé. De plus, au contraire de l'auteur des Conjectures académiques, pour qui l'origine orale des poèmes homériques expliquait et excusait leurs innombrables défauts, qu'Homère les eût chantés était pour lui la raison même

4. Sur l'évolution de la pensée de Vico sur Homère entre la première et la deuxième édition de la Scienza nuova, voir Haddock (1979).

5. Michelet a traduit l'édition de 1744, sous une forme un peu abrégée (Vico 1827 [1744]) ; c'est cette traduction que j'ai consultée.

6. Les écrits de Rousseau sur la langue et la musique seront cités d'après le tome $\mathrm{V}$ de la Bibliothèque de la Pléiade, noté ci-après $O . C$., $V$. Le tome I, cité une fois, sera noté $O . C$., $I$. 
d'une beauté qui nous enchantait encore. L'Anglais Robert Wood disait des choses fort semblables dans un ouvrage dont il rédigea les versions successives à peu près au moment où Rousseau achevait son Essai sur l'origine des langues: bien qu'Homère eût ignoré l'écriture, il le tenait pour « le plus clair et le plus intelligible en même temps que le plus musical et le plus harmonieux de tous les poètes " ${ }^{7}$. L'abbé d'Aubignac n'avait donc fait école qu'à demi. Les choses changèrent après que l'Allemand Friedrich Wolf eut publié en 1795 ses Prolegomena ad Homerum ${ }^{8}$. Faisant fond sur des manuscrits alexandrins que Gaspard d'Ansse de Villoison avait redécouverts à Venise et publiés en $1788^{\circ}$, il était en mesure d'affirmer que les épopées homériques étaient le fruit d'une lente élaboration scripturaire qui s'était poursuivie du VI $\mathrm{I}^{\mathrm{e}}$ siècle au $\mathrm{II}^{\mathrm{e}}$ siècle avant J.-C. Rien n'interdisait de penser qu'un aède nommé Homère en avait composé une première version; mais elle se présentait sous forme de chants épars qui s'étaient transmis de rhapsode en rhapsode jusqu'à ce que Pisistrate les fasse mettre par écrit et les assemble en un tout cohérent que les grammairiens ne cessèrent par la suite de retoucher jusqu'à l'époque alexandrine et au-delà. Nous étions loin de l'abbé d'Aubignac puisque son compilateur faisait place à un long cortège de scribes. En revanche, malgré les apparences, nous n'étions pas si loin de Rousseau et de Wood. Le philologue allemand les citait tous les deux avec faveur, et reproduisait même en note l'intégralité du chapitre VI de l'Essai sur l'origine des langues ${ }^{10}$ : après tout, ne devait-il pas leur savoir gré d'avoir remis en honneur l'idée que, quand un aède de la Grèce archaïque eût été à l'origine de l'Iliade et de l'Odyssée, il ne pouvait les avoir écrites.

\section{Parry et Lord, ou l'ambiguë résurrection d'Homère}

C'est que tous ces auteurs se seraient accordés sur la proposition: "Homère ne peut avoir écrit les poèmes que la tradition lui attribue ». Mais chacun d'eux la faisait suivre d'une clausule différente. Pour l'abbé d'Aubignac, c'était: "puisqu'il n’a “jamais été" "; pour Vico, c'était: "puisque Homère n'est qu'un nom, le nom d'un auteur innombrable»; pour Rousseau et Wood, c'était " puisqu'il n'a point été écrivain»; quant à Wolf, il n'ajoutait rien, et portait son attention sur le seul processus

7. «[...] the most clear and intelligible, as well as the most musical and harmonious, of all Poets " (Wood 1973 [1775] : 292).

8. On a utilisé la traduction anglaise parue en 1985.

9. Sur ce point, voir Canfora (1999), Rossi (1999).

10. Dans la traduction anglaise, l'auteur parle de la brilliant audacity de Wood et cite Rousseau comme this very clever man (voir Wolf 1985 [1795] : 71 et 103, note 54). L'abbé d'Aubignac, que Wolf devait trouver par trop désinvolte, est mentionné sans beaucoup d'aménité (Ibid. : 117, note 84). 
scripturaire qui avait produit ces poèmes. Dans tous les cas, Homère en tant qu'écrivain avait disparu de leur univers. Rousseau et Wood se distinguaient cependant en ce qu'ils croyaient malgré tout en l'existence d'un homme ayant porté ce nom. Mais le monde où sa voix avait résonné devenait plus lointain, plus inaccessible encore que celui où leurs devanciers avaient jusque-là situé Homère. Nulle trace, en effet, n'en subsistait car ce n'est pas de ce monde-là que provenaient les textes prétendument homériques qui étaient parvenus jusqu'à nous. Et pourtant, ils voulaient accéder à ce monde, ne serait-ce qu'en pensée, ou en rêve. C'est de cette quête que le présent article veut parler. Je ne suivrai pas l'ordre chronologique car je crois utile de commencer par dire un mot de l'ouvrage qui marque, en 1928, l'aboutissement du processus dont leurs réflexions et leurs rêveries posaient les premiers jalons. Cette année-là, en effet, un jeune homériste nommé Milman Parry soutint en Sorbonne une thèse intitulée: L'Épithète traditionnelle dans Homère (Milman Parry 1928). Il y montrait de façon convaincante que les artifices de diction utilisés dans le texte homérique (il les appelait des "formules») composaient un système trop complexe et trop parfaitement agencé pour avoir été forgé par un auteur unique. On devait donc y voir le produit d'une longue évolution au cours de laquelle les aèdes avaient modifié à chaque génération ce qu'ils recevaient de leurs devanciers. Autrement dit, la texture même des vers homériques laissait transparaître que des aèdes innombrables avaient contribué à leur composition. Parry devenait ainsi ce qu'un commentateur a appelé le Darwin des études homériques: l'un avait expliqué l'apparition des espèces sans recourir à l'hypothèse d'un dieu créateur, l'autre expliquait celle de l'Iliade et l'Odyssée sans recourir à l'hypothèse d'un poète créateur ${ }^{11}$. Parry ne proclamait pas, comme l'abbé d'Aubignac, qu'Homère n'avait pas existé, mais considérait simplement que, qu'il eût existé ou non, son œuvre n'était pas l'expression de son seul génie. Intuition qu'il avait déjà faite sienne dans un devoir universitaire antérieur de cinq ans à sa thèse : "La poésie [homérique] ", y avait-il écrit, "n'est pas une poésie où un poète aurait utilisé ses propres mots et tenté d'exploiter au mieux les ressources de la prosodie, mais une poésie qui au cours des siècles avait accumulé ces ressources, tous les tours de langage, tous les mots, les expressions, et les effets de position qui avaient plu à la race ${ }^{12}$. Savait-il qu'il retrouvait là les idées, et presque les mots, de Vico ?

11. Wade-Gery (1952), cité in Lord (1991: 40).

12. "The [homeric] poetry was not one in which a poet must use his own words and try as best he might to utilize the possibilities of the metre. It was a poetry which for centuries had accumulated all such possibilities - all the turns of language, all the words, phrases, and effects of position, which had pleased the race" (Milman Parry 1971 [1923] : 425). 
Parry ne se prononçait pas dans sa thèse sur le mode de composition, oral ou écrit, de l'Iliade et de l'Odyssée. Mais il acquit par la suite la conviction que les artifices formulaires qu'il y avait mis au jour étaient l'indice d'une composition orale, hypothèse qu'il entreprit de tester en enquêtant dans une région où la tradition épique vivait encore. Il se rendit donc en Yougoslavie au début des années 1930, et là, il collecta des épopées qui lui parurent comparables, par la diction et pour certaines par l'ampleur, à l'Iliade et à l'Odyssée. Des bardes le plus souvent illettrés y recouraient à des formules héritées d'une longue tradition, ils prétendaient même restituer fidèlement ce qu'ils avaient reçu de leurs devanciers - même si, en réalité, leurs chants variaient notablement d'une exécution à l'autre. Il lui était maintenant permis d'imaginer que les épopées homériques avaient été prises sous la dictée d'un barde semblable à cet Avdo Mededovic dont lui et son assistant Albert Lord transcrivaient les chants, longs eux aussi de plusieurs milliers de vers. Adam Parry, le fils de Milman, a bien dit l'émotion que son père dut alors ressentir : "C'était, contre tout espoir, notre rêve qui devenait réalité : Homère chantait, et nous pouvions le voir et l'entendre! On ne peut douter que cette idée habitait l'esprit rigoureux mais ardent de Parry lui-même ${ }^{13}$. Parry et Lord vivaient donc une scène qu'ils étaient en droit de penser semblable à une autre scène, survenue quelque vingt-cinq siècles plus tôt; ils assistaient par procuration à la mise par écrit de l'Iliade et de l'Odyssée, ils entendaient la voix d'Homère. Plus encore, de cette antique scène qui soudain ressuscitait devant eux, ils n'étaient pas seulement spectateurs mais acteurs: "non seulement", continue Adam Parry, "nous pouvions [...] voir et entendre Homère chanter, mais nous pouvions même être son scribe, nous pouvions faire ce que fit un jour l'homme qui le premier mit l'Iliade par écrit ${ }^{14}$.

Si Parry et Lord n'étaient pas les premiers à juger probable que les épopées homériques eussent été composées oralement, ils se séparaient de leurs devanciers sur plusieurs points. Au contraire de l'abbé d'Aubignac, ils voyaient en elles l'œuvre d'un aède unique plutôt qu'une rhapsodie. Et, s'ils étaient d'accord avec Wolf pour penser que plusieurs générations d'ouvriers avaient contribué à leur composition, ces ouvriers étaient pour eux les aèdes qui avaient précédé Homère tandis qu'ils étaient pour Wolf les grammairiens qui s'étaient succédé depuis Pisistrate. De plus, alors que

13. "It [was], against all hope, our dream come true : we can see and hear Homer sing! There is no doubt that such a dream animated the ardent though precise mind of Parry himself" (Adam Parry $1966: 179)$.

14. "not only [...] can we see and hear Homer sing; we can even be his scribe, we can do what the first man who put the Iliad into writing once did" (Ibid.: 183). 
personne avant eux n'aurait su dire au juste comment on était passé d'un chant oral aux textes écrits que les Anciens nous ont légués, ils se pensaient en mesure de proposer un scénario plausible. Milman Parry ne survécut à sa découverte que de quelques années, et c'est à son assistant qu'il revint de poursuivre son œuvre. Et plus abruptement que Parry ne l'eût sans doute fait, Lord tint pour acquise «l'équivalence essentielle du barde yougoslave et de l'auteur de l'Iliade» (Adam Parry 1966 : 179).

Il faut retracer ici la démarche de Lord, avec toute sa rigueur et ce qu'il faut bien appeler sa raideur. Comme Parry l'avait fait dans sa thèse pour les épopées homériques, il détailla dans son Singer of Tales les procédés de diction utilisés par les bardes yougoslaves. Et il constata que, si chacun d'eux s'en tenait à un stock de formules auxquelles il restait fidèle, les formules variaient sensiblement d'un barde à l'autre (Lord 1960 : 53). L'uniformité des formules que son maitre Parry avait repérée dans le texte homérique lui parut donc une preuve définitive que l'Iliade et l'Odyssée avaient été l'œuvre d'un aède unique : ce qui n'avait été pour Parry qu'une hypothèse devenait là une certitude que d'autres constatations étaient censées corroborer. Ainsi, les particularités formulaires présentes aussi bien dans les compositions des bardes illettrés que dans les épopées homériques paraissaient absentes des rares - et fort médiocres - compositions écrites que Parry et lui avaient recueillies en Yougoslavie : preuve supplémentaire à ses yeux que l'écriture n'avait joué aucun rôle dans la composition de l'Iliade et de l'Odyssée. Par ailleurs, les épopées qu'ils avaient prises sous la dictée différaient de celles qu'ils avaient enregistrées dans des conditions proches de celles d'une performance réelle. Les bardes qui dictaient leurs chants vers à vers ne travaillaient pas dans l'urgence comme leurs confrères. Rien ne les empêchait de donner toute l'ampleur désirable aux passages ornementaux dont ils étaient si fiers et que l'agitation ou l'impatience des auditeurs les contraignaient souvent à abréger lorsqu'ils se produisaient devant leur public habituel ; ils avaient toute latitude pour enrichir leur récit et en multiplier les péripéties sans crainte de lasser ; ils pouvaient accumuler les métaphores dont l'urgence de l'exécution les obligeait d'ordinaire à être plus économes. Or ces traits, qui distinguaient effectivement les compositions dictées par Avdo de tous les autres chants du corpus yougoslave, n'étaient-ils pas précisément ceux que la tradition s'est toujours plue à relever chez Homère? Pour Lord, le doute n'était plus permis: l'Iliade et l'Odyssée étaient des oral-dictated texts. La longueur même des poèmes d'Homère, affirmait-il, est la meilleure preuve qu'ils sont les produits d'une dictée plutôt que d'une exécution. L'ampleur de leur tempo, la plénitude de leur phrasé (the fullness of their telling), sont aussi des indices que c'est là la méthode qui a été employée (Lord 1991 : 46). 
Lord a consacré une page étonnante aux séances de dictée que lui et Parry ont organisées, longues parfois de plusieurs jours (Ibid.). Elles y prennent la figure d'une scène typique, qui ne serait ni d'aucun temps ni d'aucun lieu. Il n'y parle pas d'Avdo ni de tel autre barde mais $d u$ "poète oral " (oral poet), tant il va de soi pour lui que les observations faites en Yougoslavie valent pour les poètes oraux de tous les temps, et en particulier pour celui qui dicta un jour l'Iliade et l'Odyssée. Lord s'y souvient d'Avdo, mais il voit un aède de la Grèce archaïque. Nous pouvons partager sa vision intérieure : il nous suffit pour cela de récrire cette page en lui apportant juste les retouches nécessaires à faire apparaître non ce dont il se souvenait, mais ce qu'il voyait. Et la scène s'anime alors devant nous. Nous voyons un homme assis face à un aréopage de scribes silencieux et attentifs; comme nous ne savons pas son nom, rien ne nous empêche de l'appeler "Homère", et de baptiser "Pisistrate", "Hipparque » ou bien "Lycurgue » celui qui a dépêché ces scribes auprès de lui. L'homme a d'abord hésité car la situation est insolite pour lui, habitué qu'il est à s'accompagner d'un instrument qui puisse lui donner le tempo. Il a aussi été un peu décontenancé d'avoir à attendre après chaque vers que les scribes l'aient recopié, car son esprit va plus vite que leur stylet. Mais la bienveillance de son auditoire l'a mis peu à peu en confiance; cette technique de la dictée, au début si déconcertante pour lui, il a commencé à en sentir tous les avantages; un rythme s'est installé dans son esprit ; tout le jour il a déclamé ses hexamètres, il a recommencé le lendemain, puis le surlendemain encore... Et le monde a reçu l'Iliade et l'Odyssée. Sans doute avait-il chanté en bien d'autres occasions, mais comme les aèdes recréaient à chaque exécution un chant nouveau, ces chants disparus à jamais n'étaient pas les mêmes que ceux qu'il avait dictés en ces jours-là. Auteur de l'Iliade et de l'Odyssée, Homère ne l'avait donc été qu'à une unique occasion. Ces pages de Lord, je les intitulerais volontiers : "Les jours où Homère fut véritablement Homère ». Tel aura été le paradoxe de l'entreprise de Parry et Lord. Commencée comme la mise à mort du poète créatif, elle aboutit à un portrait du poète au travail. L'auteur que l'abbé d'Aubignac, Wolf et même le Parry de L'Épithète traditionnelle avaient fait disparaître, était donc de retour. Il n'était plus un écrivain, bien sûr, mais il restait un auteur. Non point un auteur anonyme et multiple comme le pensait Vico, mais un homme qu'on pouvait, d'une certaine manière, voir et surtout entendre.

Ce portrait a séduit bien des commentateurs, d'autres sont restés sceptiques. Ne parlons pas de la faille que comporte la construction de Parry et Lord. Ils semblent avoir considéré que le texte un jour dicté par leur 
Homère nous est parvenu tel quel ${ }^{15}$. Eux qui avaient si éloquemment montré que le texte de l'Iliade et l'Odyssée avaient eu une préhistoire orale ont curieusement oublié d'en considérer l'histoire - cette histoire que Wolf s'était précisément attaché à retracer. Mais parlons seulement de ceux qui se sont situés dans la même perspective qu'eux. Bien que Kirk fût d'accord avec Parry et Lord pour penser que les épopées homériques avaient été composées oralement, elles lui paraissaient d'une qualité trop supérieure aux chants yougoslaves pour avoir été le fruit d'une simple séance de dictée. Il fit l'hypothèse qu'à une époque où la Grèce ignorait encore l'alphabet, un aède particulièrement doué avait composé des épopées d'une taille bien plus majestueuse que celles dont ses confrères s'étaient jusque-là contentés; cette " composition monumentale» (monumental composition [Kirk 1999: 190]) avait ensuite été transmise oralement durant plusieurs générations avant d'être mise par écrit.

Adam Parry proposa une hypothèse qu'on peut dire symétrique. S'il accordait volontiers à son défunt père que l'Iliade et l'Odyssée étaient le produit d'une culture encore largement orale, il y discernait aussi la trace d'un travail de plume. La médiocrité des quelques épopées écrites que Lord et son père avaient recueillies en Yougoslavie ne lui paraissait pas une raison suffisante pour exclure une telle éventualité. On les devait, en effet, à des hommes fraîchement alphabétisés, qui tentaient maladroitement d'imiter la prose des journalistes ou des maîtres d'école. Pensons plutôt à un monde grec où l'alphabet viendrait seulement d'apparaître. Et dans ce monde encore vierge d'écriture, imaginons un barde talentueux qui déciderait d'utiliser les magiques sèmata nouvellement empruntés aux Phéniciens. Le poème qu'il écrirait aurait la même diction, la même ampleur que ceux qu'il avait chantés jusque-là puisqu'il ne connaittrait aucune autre forme de poésie. Mais il se donnerait le temps de choisir ses mots, d'amplifier sa narration et de camper ses personnages (Adam Parry 1966: 214) ${ }^{16}$. Homme d'écriture, on voit que l'Homère d'Adam Parry ne l'est que de bien étrange façon: il est l'homme d'une première écriture. Il sait écrire mais il ignore encore ce qu'est la littérature puisque le livre qu'il s'apprête à écrire sera le premier jamais écrit en terre grecque. L'Homère imaginé par Lord est lui aussi un chanteur d'une sorte particulière. Il n'est pas sorti des limites de la technique orale, mais c'est la présence de scribes autour de lui qui lui permet d'en magnifier les possibilités. D'un côté un homme d'écriture dont les seuls modèles sont oraux, de l'autre un homme de parole à qui des gens

15. C'est encore ce que semble présupposer un homériste récent : voir Bouvier (2002: 227); et aussi Minchin (2001).

16. Voir aussi Adam Parry (1956). 
d'écriture donnent la possibilité d'atteindre le meilleur de son art. L'un et l'autre appartiennent à un monde qui ne leur survivra pas. L'Homère chanteur de Lord use de techniques à lui léguées par des générations de devanciers, mais la technique nouvelle qui commence à se répandre en Grèce va bientôt les faire disparaître. C'est du moins ainsi que Lord voit les choses, au contraire, par exemple, d'un Gregory Nagy qui pense pour sa part que, jusqu’à l'époque alexandrine, et alors que des épopées écrites circulaient déjà depuis longtemps, des aèdes ont continué à en composer de nouvelles ${ }^{17}$. Mais l'Homère écrivain d'Adam Parry est lui aussi le dernier de son espèce, car dès qu'il aura écrit son livre, le monde où il vit cessera d'être un monde sans livres. Ses successeurs, eux, sauront ce qu'est la littérature. La scène imaginée par Adam Parry aussi bien que celle qu'a composée Lord sont donc à la fois primitives et ultimes.

Au moins Milman Parry et Lord ont-ils pu ressusciter pour un instant le monde où résonna la voix d'Homère; mais ils ont du même coup ressuscité son ambiguïté. Car ils savaient bien que, tout comme l'homme qui dicta un jour l'Iliade et l'Odyssée, les bardes qui leur dictaient leurs épopées n'auraient pas de successeurs. Et ces bardes eux-mêmes en étaient conscients. Quand Parry leur demandait lesquelles étaient les meilleures, des épopées chantées ou de celles qu'ils dictaient vers à vers, leur réponse était invariablement: "les épopées chantées sont plus vraies, les épopées dictées sont plus belles" (Lord 1991: 47). C'est donc qu'ils n'étaient pas dupes : la beauté qu'ils faisaient naître se payait d'un peu de fausseté, signe que leur art n'était plus vraiment celui qu'ils avaient reçu de leurs pères. Adam Parry était en revanche incapable de ressusciter son Homère. Son raisonnement l'obligeait, en effet, à supposer que, lorsque les Grecs avaient emprunté aux Phéniciens leur alphabet, ils ne leur avaient rigoureusement rien emprunté de plus (Adam Parry 1966: 214). Or il savait que, dans les pays où l'écriture était en train de se répandre à l'époque où il publiait ces lignes, son introduction allait de pair avec beaucoup d'autres emprunts. Vérité historique dont Claude Lévi-Strauss (1955: 319) a donné une version littéraire dans la "Leçon d'écriture ", ce joli conte philosophique où des Nambikwara auxquels il prêtait sa propre mélancolie étaient censés avoir compris "confusément que l'écriture et la perfidie pénétraient chez eux de concert».

Si ambiguë qu'ait été l'aventure des deux homéristes américains, je crois que la félicité y aura été finalement la note dominante. Lisons ce que Lord écrit de sa dernière entrevue avec Avdo Mededovic. Il était revenu le voir en 1951, bien après la mort de Parry, et le barde, âgé et malade, avait 
accepté de lui donner une nouvelle version du Chant de Beciragic Meho qu'il lui avait déjà dicté vingt ans plus tôt. C'était un chant qu'il avait appris dans sa jeunesse, auprès d'un chanteur nommé Mumin qui luimême le tenait d'un homme dont tous les bardes entretenaient le pieux souvenir, Cor Huso :

"Le chant s'éleva, inaltéré comme autrefois. Mais son essence était d'un temps bien antérieur à Avdo, à Mumin et à Cor Huso. Car plus de la moitié de l'histoire se déroule pendant la scène d'assemblée qui en constitue l'ouverture, et où Beciragic Meho dit les errances et les aventures, les épreuves et les souffrances qui l'ont mené à l'état misérable où il se trouve. Pour ceux qui ont des oreilles pour entendre, c'est Homère lui-même qui chante là, et parle d'Ulysse contant dans le palais d'Alcinoos les errances et les malheurs qui l'ont jeté sur la grève de la Phéacie " ${ }^{18}$.

Les auditeurs d'Avdo savent, s'ils ont des oreilles pour entendre, que c'est Homère qui chante en réalité devant eux... Il est difficile d'être plus clair - et plus candide. Ce que l'évocation des séances de dictée ne faisait que sous-entendre s'exprime ici dans le ravissement. La félicité dont ces lignes frémissent est d'une sorte dont notre littérature offre quelques exemples. Je pense à Nerval, lui toujours si anxieusement avide d'entendre dans le présent l'écho des jours enfuis, lui qui s'émerveillait en abordant le sol grec, à la vue de scènes "où le passé renaît sous l'enveloppe du présent " (Nerval 1950: I, 235). Je pense aussi à ce que le narrateur du Temps retrouvé éprouve soudainement dans la cour de l'hôtel Guermantes. Son trébuchement sur une dalle lui a remis en mémoire un incident semblable, oublié depuis longtemps. S'interrogeant sur la cause de la félicité qui s'empare alors de lui, il a ces mots qui méritent d'être mis en regard de ceux de Lord:

"Or cette cause, je la devinais en comparant ces diverses impressions bienheureuses et qui avaient entre elles ceci de commun que je les éprouvais à la fois dans le moment actuel et dans un moment éloigné, jusqu’à faire empiéter le passé sur le présent, à me faire hésiter à savoir dans lequel des deux je me trouvais; au vrai, l'être qui goûtait en moi cette impression la goûtait en ce qu'elle avait de commun dans un jour ancien et maintenant, dans ce qu'elle avait d'extra-temporel » (Proust 1954 : III, 871).

Lord a vu, lui aussi, le passé empiéter sur le présent, lui aussi a vécu une minute, "affranchie de l'ordre du temps " (Ibid. : 873), durant laquelle le monde avait cessé d'être en deuil d'Homère.

18. "From the past the song was unwound and the tale emerged. Its essence, however, was from a time long before Avdo and Mumin and Cor Huso; for more than half of this song takes place in the assembly with which it opens, as Beciragic Meho tells of his wanderings and adventures, his trials and sufferings which have brought him to the present sorry state. To those who have ears to hear, Homer is singing of Odysseus in the court of Alcinous, recounting his wanderings and the misfortunes which had brought him to the shores of Phaeacia" (Lord $1991: 71$ ). 


\section{Rousseau ou le deuil d'Homère}

344 D'autres avant lui avaient porté le deuil d'Homère, et sans trouver de consolation. C'est ici qu'il faut revenir à Jean-Jacques Rousseau. Considérons en effet le rôle que son Essai sur l'origine des langues fait jouer à Homère. Rédigé sous sa forme finale entre 1756 et $1761^{19}$, publié en 1781 au tome III des CEuvres posthumes de J.-J. Rousseau, l'ouvrage se compose de vingt chapitres le plus souvent assez brefs, dont la rapide succession donne à l'argumentation la trame serrée qu'on retrouve si souvent chez Rousseau. Au chapitre IV, l'auteur imagine les caractères originaux qui, si elle existait encore, distinguerait la première langue de toutes les autres. Sensuelle plutôt qu'exacte, «elle auroit beaucoup d'irrégularités et d'anomalies, elle négligeroit l'analogie grammaticale pour s'attacher à l'euphonie, au nombre, à l'harmonie, et à la beauté des sons » (O.C., $V: 383)$; les synonymes y abonderaient et les tours y «devroient être en images, en sentiments, en figures». Mais ce sont surtout les particularités de sa "partie mécanique» qui intéressent Rousseau :

"Comme les voix naturelles sont inarticulées, les mots auroient peu d'articulations [...]. En revanche les sons seroient très variés, et la diversité des accens multiplieroit les mêmes voix : La quantité, le rhythme seroient de nouvelles sources de combinaisons; en sorte que les voix, les sons, l'accent, le nombre, qui sont de la nature, laissant peu de chose à faire aux articulations qui sont de convention, l'on chanteroit au lieu de parler».

Pauvre en consonnes, elle se réduirait donc pour l'essentiel à des voyelles de timbre, de hauteur, d'intensité et de durée variables, qui sortiraient naturellement du gosier sans être altérées par des modifications de la langue ou du palais. Comme l'a remarqué Catherine Kintzler, les propriétés de cette langue originelle évoquent celles que l'Émile attribue au langage du nourrisson $(2006: 371)$. Il me semble qu'elle fait aussi penser aux vocalises de nos chanteurs, ces fluides émissions sonores dont les consonnes sont bannies. Pour ce qui est de la fluidité, je suis sûr que Rousseau s'est souvenu de ce que sa Lettre sur la musique françoise disait en 1753 sur la douceur de l'italien, où les articulations sont "peu composées ", où " la rencontre des consonnes [...] est rare et sans rudesse ", et où « un très-grand nombre de syllabes n’y étant formées que de voyelles, les fréquentes élisions en rendent la prononciation plus coulante" $(O . C$., $V:$ 297). Quels que soient cependant les souvenirs auditifs qui lui ont inspiré ses méditations, ils se sont certainement mêlés à des souvenirs livresques car il n'était pas le premier à spéculer sur la langue originelle et

19. Sur la datation de l'Essai et des textes préparatoires, voir notamment Duchez (1974) et Kintzler (1993). 
à supposer qu'elle "participait du chant ${ }^{20}$. Du reste, cette langue originelle, comme d'ailleurs l'état de nature dans le second Discours, est pour lui un pur être de raison, une forgerie philosophique ${ }^{21}$ que son raisonnement lui imposait de tenir à distance de toutes les langues qu'il avait eu l'occasion d'entendre parler. Les trois seules langues que l'Essai rapproche d'elle ne sont parlées que par des peuples éloignés dans le temps ou dans l'espace : ce sont le chinois, l'arabe et le grec ancien. Comme on peut s'en douter, le chinois est mentionné parce qu'il est ce que nous appellerions aujourd'hui une langue à tons: alors que "les notes de la musique", qui "sont autant d'accens", ne sont utilisées qu'en petit nombre dans nos langues, "les Chinois en ont beaucoup davantage; en revanche ils ont moins de consonnes" (O. C., $V: 382)$. Et l'arabe intervient non pour sa partie mécanique, mais parce qu'il «a plus de mille mots différens pour dire un chameau, plus de cent pour dire un glaive. Etc." (Ibid.).

Quant au grec, les raisons de sa présence dans cette énumération n’apparaissent qu'au chapitre XII : "Une langue qui n'a que des articulations et des voix n'a donc que la moitié de sa richesse ; elle rend des idées, il est vrai, mais pour rendre des sentimens, des images, il lui faut encore un rhythme et des sons, c'est-à-dire une mélodie : voilà ce qu'avoit la langue grecque, et ce qui manque à la nôtre " (Ibid. : 411). Comprenons qu'une langue où l'on ne peut comme en grec jouer sur la longueur et la hauteur des syllabes ne rend que des idées, car les consonnes et les voyelles seules ne suffisent pas à exprimer les sentiments. C'est là encore une opinion très répandue à l'époque, et Rousseau n'a cessé d'en proposer de nouvelles formulations. On lit au détour d'un manuscrit où il esquissait dès avant 1755 les principaux thèmes de l'Essai ${ }^{22}$ : " de toutes les langues connues la Grecque étant sans difficulté celle qui avoit le plus de résonance et d'accent, il s'ensuit que c'est aussi celle de toutes où le discours doit être le plus semblable au chant». Vingt ans plus tard, les Fragmens d'observations Sur l'Alceste italien de M. le Chevalier Gluck ${ }^{23}$ décrivent encore le grec comme une langue "vraiment harmonieuse et musicale", qui «avoit par elle-même un accent mélodieux». Et il en est de même

20. Le mot est de Condillac dans l'Essai sur l'origine des connaissances humaines (cité in Cannone 1990 : 49). La question se pose également de savoir ce que Rousseau doit ici à Vico, et il est difficile de la trancher : voir Derrida (1967: 381); Tomlinson (1999: 375-376, note 25).

21. À vrai dire, le statut de la langue originelle dans l'Essai - réalité ou fiction - est peut-être plus ambigu que celui de l'état de nature dans le second Discours (voir la belle analyse de Guetti 1969).

22. O. C., $V: 333$. Sur la datation de ce manuscrit, édité sous le titre L'Origine de la mélodie, voir Duchez (1974).

23. O. C. 445. Sur la datation de ce texte, noté Fragmens par la suite, voir Olivier Pot (1995), in O. C., $V$ : CCXIII. 
dans l'article «Voix » du Dictionnaire de musique auquel il a travaillé entre 1749 et $1764^{24}$ :

«Dans une langue qui seroit toute harmonieuse, comme étoit au commencement la Langue Grecque, la différence de la Voix de parole à la Voix de chant serait nulle; on n'auroit que la même Voix pour parler et pour chanter ; peut-être est-ce encore aujourd'hui le cas des Chinois" (O.C., $V: 1149)$.

La terminologie peut paraître flottante, mais l'idée que Rousseau a en tête n'a pas varié, et c'est finalement au chapitre XII de l'Essai qu'elle est le plus clairement exprimée : la langue grecque a "un rythme et des sons", autrement dit les syllabes s'y distinguent comme longues ou brèves, et la hauteur des sons y est variable. Rousseau et ses contemporains attribuaient, en effet, une très grande amplitude aux variations de hauteur dans le grec ancien. Ainsi, Duclos avait cru pouvoir s'appuyer sur Denys d'Halicarnasse pour affirmer dans une de ses Remarques sur la Grammaire de Port-Royal que "l'élévation du ton dans l'accent aigu et l'abaissement dans le grave étoient d'une quinte ${ }^{25}$. Rousseau cite cette remarque au chapitre XII de l'Essai sans la contredire, et il semble s'en être encore souvenu dans l'article "Opéra " du Dictionnaire de musique, où on lit: "on ne sauroit entendre les passages des Grecs sur leur manière de réciter ", y écrit-il, "qu'en supposant leur Langue tellement accentuée que les inflexions du discours dans la déclamation soutenue, formassent entr'elles des Intervalles musicaux et appréciables " (Ibid. : 949). Il n’est certes plus question de quintes, mais tout de même d'intervalles que l'oreille peut évaluer, qu'un instrument peut reproduire, et qu'un musicien pourrait transcrire sur une portée. Et lorsqu'il suggère dans l'article "Tétracorde" qu'en divisant leur système musical par tétracordes et non par octaves comme nous le faisons, les Grecs avaient tâché de rendre «par des Intervalles plus serrés les inflexions de voix que leur langue sonore et harmonieuse donnoit à leur récitation soutenue, et surtout à celle de leur Poésie, qui d'abord fut un véritable Chant" (Ibid.: 1119), nous retrouvons, sinon la quinte, du moins la quarte, puisque c'était en général l'ambitus des tétracordes grecs. Un intervalle d'une quarte entre les graves et les aigus, voilà qui ferait effectivement une langue fort musicale ${ }^{26}$.

24. Sur cette datation, voir Eigeldinger (1995) et Robinson (1984: chap. I). Les articles où ce thème est le plus développé sont : "Mesure ", "Opéra ", "Récitatif ", "Rythme» et "Voix ».

25. Cité in O.C., $V: 391$. En fait, les éditeurs font remarquer que l'écrivain grec avait seulement dit que la mélodie du langage parlé " est mesurée par un seul intervalle qui vaut à très peu près ce qu'on nomme une quinte" (Ibid. : 1556-1557, note 1).

26. La chose ne serait d'ailleurs pas invraisemblable en soi. Junzo Kawada signale que, chez les Mosi du Burkina-Faso, le registre courant de la voix s'étend sur un intervalle d'une quinte environ (Kawada 1998 : 14). 
Je ne crois pas que Rousseau se contente là de sacrifier aux idées du temps car, pour le théoricien de la musique et le compositeur qu'il a été, la musicalité du grec ancien était vraiment une affaire personnelle. "C'est un grand et beau problême à résoudre ", écrit-il ainsi dans les Fragmens, « de déterminer jusqu’à quel point on peut faire chanter la langue et parler la Musique. C'est d'une bonne solution de ce problême que dépend toute la théorie de la Musique Dramatique » $(O . C$., $V: 445)$. Ce problème est double. Il faut d'une part que la musique des airs s'accorde aux paroles. Sur ce point, on sait que la préférence du théoricien est toujours allée à l'italien. Ce qui n'a pas empêché le compositeur de le contredire en quelque sorte : s'il a mis en musique quelques pièces italiennes et composé des motets en latin, son œuvre maîtresse reste Le Devin du village, opéra de style bien français où les contemporains charmés saluèrent «les rapports heureux de la Musique avec les paroles » ${ }^{27}$. D'autre part, comme un opéra réduit à une suite d'airs ennuierait le spectateur, il «faut couper et séparer les Chants par de la parole ; mais il faut que cette parole soit modifiée par la Musique » ${ }^{28}$. D'où la nécessité de cette voie moyenne qu'est le récitatif, et là, la position de Rousseau sur l'italien a évolué au cours des années. Aussi bien pour le récitatif que pour le chant, la Lettre sur la musique française lui accordait par polémique toutes les faveurs qu'elle refusait au français. Encore qu'il s'y souciait aussi de détailler ce que devrait être un récitatif adapté à la langue française, conseils dont Gluck saurait plus tard faire son profit. Le Dictionnaire de musique est plus nuancé ${ }^{29}$ : même si l'italien y reste préféré au français, certains récitatifs italiens y sont critiqués; et la langue donnée comme la plus naturellement appropriée au récitatif est le grec: "Chez les Grecs", écrit-il à l'article "Récitatif ", "toute la Poésie étoit en Récitatif, parce que la Langue étant mélodieuse, il suffisoit d'y ajoûter la Cadence du Mètre et la Récitation soutenue, pour rendre cette Récitation tout-à-fait musicale; d'où vient que ceux qui versifioient appelloient cela chanter" (Ibid.: 1008). Jugement qui fait contraste avec ce que l'Essai dit de l'italien : "La langue italienne non plus que la françoise n'est point par elle-même une langue musicale. La différence est seulement que l'une se prête à la musique, et

27. La formule apparaît dans un article publié par le Mercure en avril 1777, et, à la même époque, La Harpe écrivait le même genre de chose à un correspondant. Ces textes (cités par Olivier Pot in O.C., $V$ : CCXX) sont tardifs mais ils expriment des opinions qui se sont manifestées dès la création de la pièce. Et Rousseau, pour une fois d'accord avec ce qu'on pensait de lui, fait vanter par l'un des protagonistes de ses Dialogues le «parfait accord des paroles et de la musique » qui se fait sentir dans son Devin (O. C., I: 683).

28. Article "Récitatif " du Dictionnaire de musique (O.C., V: 1008).

29. Sur l'ambivalence des vues musicales exprimées dans le Dictionnaire de musique, voir Verba (1989). 
que l'autre ne s'y prête pas »(O.C., $V: 392)$. La langue grecque, que son inaccessibilité parait de vertus auxquelles même l'italien ne pouvait prétendre malgré toute la dilection qu'il lui portait, aura donc été pour Rousseau la langue où un problème qui tourmenta toute sa vie de musicien ne se posait pas. À moins qu'il n'y ait quelque chose de plus. Si l'on met à part quelques menues pièces écrites au soir de sa vie, sa carrière de compositeur s'est pratiquement achevée en 1752 ou 1753, qui furent à la fois les années où le Devin du village la porta à son apogée, et celles où le bruit soulevé par le premier Discours l'installa sans retour dans la carrière des lettres (Tiersot 1920: 109-110). Il aura fallu que le compositeur meure en lui pour que le prosateur advienne. Cessa-t-il pour autant d'être musicien ? Disons qu'il lui arriva encore de l'être, mais par procuration, et dans le seul champ de la littérature (Pot 1995: CXXXV), à l'époque, par exemple, où il composait pour la Nouvelle Hélö̈se ces passages où le musicologue Jean-Michel Bardez croit voir de vrais récitatifs (Bardez 1980 : 54). La musicalité que Rousseau prêtait au grec, c’est celle dont sa prose portait la nostalgie.

Une fois détaillés les caractères distinctifs de la première langue, Rousseau entreprend au chapitre $\mathrm{V}$ de dire comment l'écriture contribue peu à peu à les abolir. Incapable de rendre «les sons, les accens, les inflexions de toute espèce qui font la plus grande énergie du langage ", l'écriture fait perdre en expression ce qu'elle fait gagner en exactitude. Les hommes alors ne font plus que lire en parlant; et d'une langue imprécise mais expressive parce que toute en musicalité, nous voilà passés à une langue exacte mais froide, que nous parlons comme nous l'écririons. On voit, dans ce dispositif argumentatif, toute l'importance d'Homère tel qu'il apparaît au chapitre VI, en poète qui chante et ne sait pas écrire. Il parlait une langue naturellement musicale, il n'usait pas encore d'un outil qui ne peut que dénaturer la langue; en lui, le grec avait encore retenu quelques-unes des qualités de la langue première. Les langues ensuite se sont écrites, sont devenues raisonneuses, ont exprimé des idées et non plus des sentiments, et l'Europe s'est peuplée de barbares dont les nordiques idiomes se ressentent de la rudesse des climats qui les avaient vus naître. Comme pour Lord et Parry, mais de façon encore plus dramatique que pour eux, Homère participe donc d'un monde qui va périr après lui, un monde dont le musicien Rousseau n'a cessé d'éprouver la perte.

Rousseau ne s'est pas soucié de pourtraire son Homère, ni d'en faire le héros de quelque scène de sa composition. Mais il a imaginé la naissance de la langue première. Le chapitre IV en avait établi les caractères par un raisonnement a priori; le chapitre IX évoque les jours heureux où une humanité encore innocente naquit à la parole. La scène se passe dans une 
contrée du Midi, car les langues nordiques qui deviendraient les langues des Barbares ne sont apparues que plus tard:

« [...] dans les lieux arides où l'on ne pouvoit avoir de l'eau que par des puits, il falut bien se réunir pour les creuser, ou du moins s'accorder pour leur usage. Telle dut être l'origine des sociétés et des langues dans les pays chauds.

Là se formérent les prémiers liens de familles, là furent les prémiers rendez-vous des deux séxes. Les jeunes filles venoient chercher de l'eau pour le ménage, les jeunes hommes venoient abruver leurs troupeaux [...]. Sous de vieux chênes vainqueurs des ans une ardente jeunesse oublioit par dégrés sa férocité, on s'apprivoisoit peu à peu les uns avec les autres; en s'efforçant de se faire entendre on apprit à s'expliquer. Là se firent les prémiéres fêtes, les pieds bondissoient de joie, le geste empressé ne suffisoit plus, la voix l'accompagnoit d'accens passionnés, le plaisir et le désir confondus ensemble se faisoient sentir à la fois. Là fut enfin le vrai berceau des peuples, et du pur cristal des fontaines sortirent les prémiers feux de l'amour» (O.C., $V: 405-406$ ).

L'idée est reprise au chapitre XII :

"Autour des fontaines dont j'ai parlé les prémiers discours furent les prémiéres chansons; les retours périodiques et mesurés du rhythme, les infléxions mélodieuses des accens firent naitre la poésie et la musique avec la langue; ou plustôt tout cela n'étoit que la langue même pour ces heureux climats et ces heureux temps où les seuls besoins pressans qui demandoient le concours d'autrui étoient ceux que le cœur faisoit naitre » (Ibid. : 410).

Ainsi donc, les premières langues de l'humanité seraient nées au Midi du monde, dans des fêtes innocentes et heureuses où le plaisir et le désir se disaient en accents passionnés et chantants.

Parry et Lord auront été témoins et acteurs d'une scène où ils crurent revivre des jours qu'Homère lui-même avait autrefois vécus. La scène dont Rousseau fait naître la langue première n'existe que dans son imagination, mais si fort imprimée qu'elle y acquiert une sorte de réalité, et que son œuvre en multiplie les versions. L'une est la scène de fête dont il dit avoir été le témoin à Saint-Gervais, et qu'il évoque à la fin de la Lettre sur les spectacles. Elle n'est plus alors qu'un souvenir embelli par l'éloignement, et où il serait naïf de voir le prototype des fêtes heureuses dont l'évocation parsème tous ses écrits. Disons qu'une image qui s'est formée en lui dans les souffrances de l'âge mûr mêle à des souvenirs transfigurés des aspirations faites d'abord de la négation de ce qu'il avait sous les yeux. Il en est de même, au fond, de la langue première: les souvenirs de Venise, où comme mélomane au moins il fut heureux, sont venus enrichir un rêve nourri d'abord de son malaise devant la langue futile et légère des salons parisiens où il avait tant de mal à tenir sa partie. Une autre version est la fête des vendanges au livre vi de la Nouvelle Hélö̈se. Et il y a surtout la fête villageoise par laquelle il conclut son Devin du village. C'est une fête égalitaire et populaire comme celle de Saint-Gervais; tout 
y est rustique et simple comme à Clarens ; sous les ormeaux que les nécessités de la rime lui ont fait préférer aux vieux chênes, les fillettes et leurs galants dansent au son des chalumeaux comme aux premiers temps de l'humanité parlante. Et Homère qui chante et qui ne sait pas écrire a lointainement part à ces fêtes ${ }^{30}$.

\section{Wood sur les traces d'Homère}

Robert Wood était arrivé par des voies indépendantes à des positions assez proches. Ses promenades archéologiques l'avaient conduit dans «les contrées où Achille avait combattu, où Ulysse avait voyagé, où Homère avait chanté " ${ }^{31}$. L'action de la nature et le labeur des hommes les avaient assurément transformées depuis les jours où Homère les avait contemplées, mais pas au point, lui sembla-t-il, qu'on ne puisse plus leur appliquer les épithètes dont ses chants ornaient leurs noms. C'est donc qu'en ces lieux fortunés, quelque chose du temps d'Homère subsistait encore. Et lui, marchant sur les traces du vieil aède, il les avait parcourus, il avait visité la totalité du royaume de Priam, gravi les pentes de l'Ida, remonté le Scamandre jusqu'à sa source, affronté en abordant l'Égypte des tempêtes pareilles à celles qui terrifièrent Ménélas et ses compagnons. Le deuil n'était cependant pas absent de son livre. Sur la carte de la Troade qu'il y fit tracer à partir de ses observations, il laissa en blanc le site de la ville de Troie elle-même, car il savait que déjà vingt siècles avant lui, «les ronces avaient recouvert Pergame et tout jusqu'aux ruines en avait péri " (ac tota teguntur/Pergama dumetis; etiam periere ruinae). Ce sont, empruntés à Lucain, les derniers mots de l'ouvrage.

Autant dire que ce dilettante en qui certains ont voulu voir un lointain précurseur de Schliemann aura été moins téméraire que son émule. Il était tout aussi prudent dans ce qu'il disait d'Homère. Ainsi, soucieux d'atténuer l'étonnement que les lecteurs pourraient «éprouver à voir insinuer qu'Homère ne savait ni lire ni écrire", il leur rappelait comme un "fait indubitable» que «le temps n'était pas si lointain où de grands hommes d'État et de profonds politiques ne connaissaient pas leur alphabet». Et l'idée paraîtra moins paradoxale, ajoutait-il, «si nous considérons que la poésie est le fait du génie, et l'écriture le fait de l'art ", propos qu'il amplifiait encore en une période digne de Rousseau (Wood 1973 [1775] : 247-248) :

«La poésie est déjà présente chez les Sauvages, et chez eux déjà elle n’est pas dépourvue

de ses pouvoirs magiques sur les passions qui sont les caractéristiques si vantées de ce

30. Sur les «fêtes » chez Rousseau, voir aussi Sosso (1999: 142 sqq.), Starobinski (1971 [1957] : 117), Viglieno (2001). J'achevai la rédaction de cet article, quand j'ai pris connaissance du beau livre de Michel Brix (1997), où sont avancées des idées proches de celles que développe ce paragraphe.

31. Wood (1973 [1775] : v). Sur les voyages de Wood, voir Hutton (1927). 
qu'elle est dans sa perfection. Alors que l'art merveilleux de relier l'ouïe et la vue au moyen de signes arbitraires qui n'ont d'autre rapport avec les idées qu'ils expriment que ceux que la convention leur donne, ne peut venir qu'après une profonde réflexion ».

Et même ces prudentes conjectures, il avait longtemps hésité à les livrer au public. La première version de son ouvrage se présentait comme une épître à l'un des deux amis qui l'avaient accompagné en Orient. Il était en train de la remanier pour la donner à l'imprimeur quand il fut nommé secrétaire d'État, ce qui l'obligea à renoncer pour longtemps à ses travaux d'écriture. Une dizaine d'années plus tard, en 1767 , il donna à sa lettre la forme d'une dissertation qu'il fit imprimer en quelques exemplaires destinés à ses proches: A comparative View of the antient and present state of the Troade. To which is prefixed an Essay on the Original Genius of Homer. Il n'avait pas osé y écrire qu'Homère était illettré, et n'en prit le risque que peu avant sa mort en 1769, dans une version plus longue qu'il fit imprimer sous le titre An Essay on the Original Genius of Homer, là encore en un très petit nombre d'exemplaires. Le manuscrit de cet ouvrage pratiquement inédit fut confié à l'helléniste Jacob Bryant, qui le fit paraitre en 1775. Le titre était devenu An Essay on the Original Genius and Writings of Homer: with a comparative view of the Ancient and present state of the Troade. L'ajout de and Writings montre assez que, si Bryant ne s'était pas permis de modifier le texte dont on lui avait confié le dépôt, les opinions de l'auteur sur la composition des poèmes homériques l'avaient laissé sceptique $^{32}$. Et de fait, cette vision d'un Homère illettré souleva les sarcasmes de quelques contemporains. Mais, surtout après la publication de l'Essai sur l'origine des langues en 1781 et des Prolegomena de Wolf en 1795, l'idée allait bientôt cesser de paraître blasphématoire.

Wood devait toutefois admettre que sa thèse soulevait une question: comment Homère avait-il pu, sans l'aide de l'écriture, retenir et communiquer tout ce qu'il savait ? Sans aller jusqu'à proposer un scénario détaillé comme le feraient Parry et Lord, il y répondait en invoquant des faits bien attestés qui montraient l'étendue de la mémoire orale dans les sociétés ignorant l'alphabet ${ }^{33}$ :

«Les Mexicains, qui n’avaient pas d'alphabet, et dont l'écriture idéographique sur les feuilles des arbres ne pouvait suffire à consigner les faits historiques, se fiaient à la mémoire de leurs poètes et de leurs orateurs, dont les récitals ont permis aux Espagnols

32. On a suivi ici Spencer (1957).

33. "The Mexicans, who had no alphabet, and whose picture-writing on the leaves of tress was very insufficient for the purpose of history, trusted to the memory of their Poets and Orators, from whose recitals the Spaniards wrote down the accounts which they have transmistted. In like manner the historians of Ireland have collected their materials from the lays of their Bards, and Fileas; whose accounts have been merely traditional» (Wood 1973 [1775] : 259). 
d'écrire les chroniques qui nous sont parvenues. De la même manière, les historiens de l'Irlande ont collecté leurs matériaux auprès de bardes et de voyants qui tenaient leur savoir de la simple tradition [orale] ».

Homère cessait ici d'être un cas unique pour devenir la variante grecque d'un personnage dont toutes les civilisations encore barbares pouvaient produire un ou plusieurs exemplaires. Il en était de même pour les héros de ses épopées puisque Wood affirmait que les Bédouins rencontrés au cours de ses voyages en Syrie étaient semblables, par la rudesse aussi bien que par la grandeur, aux guerriers qui s'affrontèrent sous les murs d'Ilion. Les hommes de ces pays étaient donc comme les paysages où ils évoluaient : ils n'avaient pas totalement changé depuis le temps où Homère les avait célébrés. Laède de Chios ne se fondait pas pour autant dans une foule anonyme, car parmi les bardes innombrables dont il se découvrait le frère, il en était un sur lequel on pouvait mettre un nom. Au moment où Wood écrivait l'avantdernière version de son Essay, James Macpherson venait juste de publier The Works of Ossian (1765), qui faisait suite à Fingal, An Ancient Epic (1761) et Temora, An Epic Poem (1763). Ce poète écossais disait avoir retrouvé des manuscrits gaëliques où se conservait l'œuvre d'un barde nommé Ossian que la tradition situait au III siècle de l'ère chrétienne. Ses publications lui valurent d'emblée un succès immense mais controversé : quelques commentateurs s'empressèrent, en effet, d'émettre des doutes sur l'authenticité de ces épopées prétendument ossianiques. Ces doutes, non seulement Wood ne les partageait pas, mais pour expliquer à son lecteur ce qu'avait pu être le rôle de ceux qui transcrivirent les épopées homériques, il ne croyait pas faire mieux que de les comparer à celui que "l'ingénieux éditeur de Fingal » avait joué vis-à-vis d'Ossian (Wood 1973 [1775] : 279). Il y avait là un chassé-croisé remarquable, car si Wood prêtait les traits d'Ossian à son Homère, et ceux de Macpherson à son Pisistrate, l'Écossais, à l'inverse, avait vu dans Ossian «l'Homère des Highlands » et s'était comparé lui-même à Pisistrate. En un mot, l'Ossian de Macpherson avait quelque chose d'homérique, l'Homère de Wood quelque chose d'ossianique. Et l'aller et retour devait continuer : Germaine de Staël ferait d'Ossian l'« Homère du Nord ", tandis que Wolf et ses successeurs jusqu'à Parry et Lord se souviendraient encore d'Ossian quand ils parleraient d'Homère ${ }^{34}$.

Par ailleurs, l'Homère de Wood, chanteur autant que poète, disposait comme celui de Rousseau d'une langue admirablement adaptée à la versification. Les particules du grec, pouvait-on lire dans l'Essay, « sont à l'hexamètre ce que les pierres de raccord (final stones) sont pour une pièce de

34. Sur les interférences entre la question homérique et la question ossianique, sujet immense que je ne fais ici qu'effleurer, voir Simonsuuri (1979), Haugen (1998,) Porter (2001), Dimopoulou (2006), Dué (2006). 
maçonnerie, prêtes à remplir les vides et les interstices, et à joindre entre elles les pierres de plus grande taille de façon à donner plus de lissé et de compacité au tout " (Ibid.: 287). À quoi s'ajoutaient, ce qui annonçait la thèse de Parry, les facilités que procurent des épithètes permettant "d'améliorer à la fois la sonorité et le sens, car on ne sait trop si c'est l'oreille qui est frappée par l'harmonie ou l'esprit par l'imagerie, procurée par ces épithètes composées sonores et descriptives, qui ont dans cette langue un effet inconnu de tout autre " (Ibid. : 289). Et Wood, qui situait son Homère à une époque où «le langage de la convention et de l'art n'avait pas encore prévalu sur celui de la nature et de la vérité » (Ibid. : 284) avait là encore des accents fort rousseauistes quand il prolongeait son propos d'une méditation sur l'origine du langage :

«Le langage que nous amenons avec nous dans le monde n'est pas confiné aux organes de la parole ; mais il est composé de la voix, de la contenance, et du geste. Et si la capacité à articuler qui nous caractérise ne nous avait pas suggéré un moyen plus commode d'exprimer nos idées, les simples tons de la nature, avec les variations de modulation, toutes choses qui sont aujourd'hui du domaine exclusif de la musique, auraient pu être utilisés dans la vie courante, comme c'est parait-il jusqu'à un certain point le cas en Chine. Le chant et la parole seraient deux choses peu différentes, comme les mots grecs, qui signifient à la fois l'une et l'autre chose, semblent l'impliquer » ${ }^{35}$.

On n'avait donc pas vraiment cessé, même après l'abbé d'Aubignac, d'écrire des "vies" d'Homère, ou du moins de brosser des portraits d'Homère au travail. Car les écrits que je viens de parcourir ne sont que quelques-uns parmi bien d'autres. L'infidèle traduction de l'Iliade que madame Dacier fit paraître en 1711 était accompagnée d'une vie d'Homère. Tout aussi infidèle, mais incomparablement plus belle, l'Iliade de Pope parue en 1714 était préfacée par un Essay on the Life, Writings and Learning of Homer. Vingt ans plus tard, Thomas Blackwell fit paraitre une enquête sur la vie et les écrits d'Homère, qui serait louée par toute l'Europe. Autant d'auteurs pour qui Homère était resté un écrivain, comme il l'était avant l'abbé d'Aubignac et même avant Flavius Josèphe. Ce n'est pas le cas des auteurs que $j$ 'ai retenus ici. Du coup, leur manière d'envisager la vie d'Homère ne pouvait être que très différente. Jusque-là, les biographes

35. "The Language which we bring into the world with us is not confined to the organs of speech; but it is made up of voice, countenance, and gesture. And had not our powers of articulation, that distinguishing mark of our social constitution, suggested a more convenient mode of conveying our ideas, the simple tones of Nature, with the varieties of modulation, which are now assigned to the province of music, might have been applied to the purposes of common life, as we are told they are in some degree among the Chinese. Speaking and singing would differ little, as the original Greek words, which signify both, seem to imply" (Wood 1973 [1775] : 282-283). 
d'Homère avaient composé son portrait à partir des énoncés qu'ils lui prêtaient; de ce qu'il était supposé avoir écrit, ils déduisaient ce qu'il avait pu être. Eux se sont attachés aux conditions d'énonciation des chants qui lui étaient attribués. Autant qu’à ce qu'il avait dit, ils s'étaient intéressés à la façon dont il l'avait dit ${ }^{36}$. Et pour eux, pas de doute, il avait chanté, et c'est sa voix qu'ils voulaient retrouver. Parry et Lord l'ont imaginée semblable à celle des bardes serbo-croates; Rousseau l'a imaginée d'après une musique qu'il portait en lui, celle-là même que ses lecteurs croient entendre aujourd'hui dans sa prose ; et nous venons de voir que Wood lui a également prêté une langue chantante. Par ailleurs, tous se sont accordés à lui imaginer des semblables. Pour Parry et Lord, c'est bien simple: ces semblables, ils les avaient vus et entendus; pour Wood, le semblable s'appelle Ossian; Rousseau est moins affirmatif, encore qu'il note en passant que les gondoliers de Venise chantent les poèmes du Tasse comme les rhapsodes ioniens ont dû chanter ceux d'Homère. Et tous les quatre, au contraire des modernes de la Querelle, mettent leur Homère plus haut que tous ses successeurs. La préférence pour le primitif dont Gombrichs a cru percevoir la sourde récurrence dans toute l'histoire de l'art occidental s'exprime ici sous la forme d'une préférence pour l'oralité qui a d'ailleurs perduré jusqu'à nos jours. Marcel Jousse l'a exprimée dans L'Anthropologie du geste avec l'homilétique solennité qui lui est habituelle: "Écrire, surtout dans notre milieu ethnique de Style écrit, c'est accoucher et coucher simultanément la mort sur ce linceul mortuaire qu'est la feuille de papier" (Jousse 1969: 172). Florence Dupont est plus espiègle mais guère plus optimiste quand elle écrit: "Vue du banquet homérique, notre pratique de la littérature est une culture de conserve qui cherche à donner l'illusion du frais " (Dupont 1991 : 281). Quant à Paul Zumthor, il n’a cessé de gémir de livre en livre sur la disparition des cultures de la voix. Et j’ai déjà parlé plus haut de la "Leçon d'écriture": le monde qu'on y voit périr n'a-t-il pas ce que Lévi-Strauss lui-même a appelé ailleurs la "grandeur indéfinissable des commencements» (1955: 454). Homère est l'une des figures de ces commencements. Il est aussi celui avec qui périt un monde dont nous portons le deuil. Il est le premier et le dernier.

Centre national de la recherche scientifique Centre d'études des mondes africains (CEMAF), Ivry-sur-Seine casajus@ivry.cnrs.fr

36. Rousseau s'était aussi intéressé au contenu des épopées homériques (voir Touchefeu 1995; Perrin 1999; Norman 2005), mais sans rien en déduire quant à la vie d'Homère. 
MOTS CLÉS/KEYWORDS : question homérique/homeric question - théorie orale formulaire/ oral-formulaic theory - Milman Parry- Albert Lord - Jean-Jacques Rousseau.

\section{BIBLIOGRAPHIE}

Aubignac, François Hédelin, (abbé d')

1715 Conjectures académiques ou Dissertation sur l'Iliade. Ouvrage posthume, trouvé dans les recherches d'un savant. Paris, François Fournier.

Bardez, Jean-Michel

1980 La Gamme d'amour

de Jean-Jacques Rousseau. Genève, Slatkine.

\section{Boileau, Nicolas}

1837 Euvres complètes de Boileau Despréaux. Précédées des Euvres de Malherbe. Suivies des Euvres poétiques de J.-B. Rousseau. Paris, F. Didot Frères.

\section{Bouvier, David}

2002 Le Sceptre et la Lyre.

Grenoble, Jérôme Millon.

Brix, Michel

1997 Les Déesses absentes. Vérité et simulacre dans l'ouvre de Gérard de Nerval.

Paris, Klincksieck.

Canfora, Luciano

1999 «La découverte du Venetus Marcianus A par Villoison ", in Françoise Létoublon \& Catherine Volpilhac-Auger, eds, Homère en France après la Querelle (1715-1900). Actes du colloque de Grenoble (23-25 octobre 1995), Université Stendhal-Grenoble 3.

Paris, Honoré Champion : 41-49.

\section{Cannone, Belinda}

1990 Philosophies de la musique 1752-1789. Paris, Aux amateurs de livres.

Derrida, Jacques

1967 De la grammatologie. Paris, Minuit.

Dimopoulo, Barbara

2006 «Voix et inspiration de l'aède romantique, ou quelques représentations d'Homère ", in Corinne Grenouillet
\& Éléonore Reverzy, Les Voix du peuple dans la littérature des XIXe et XXe siècles. Strasbourg, Presses universitaires de Strasbourg : 41-53.

\section{Duchez, Marie-Élizabeth}

1974 «Principe de la Mélodie et Origine des langues : un brouillon inédit de Jean-Jacques Rousseau sur l'origine de la mélodie ", Revue du musicologie 60 (1-2) : 33-86.

\section{Dué, Casey}

2006 "The Invention of Ossian", Classic@(an Online Journal) 3: The Homerizon. Conceptual Interrogations in Homeric Studies. Washington, Center for Hellenic Studies.

[http ://chs.harvard.edu/publications.sec/cla ssics.ssp.]

\section{Dupont, Florence}

1991 Homère et Dallas. Paris, Gallimard.

Eigeldinger, Jean-Jacques

1995 "Dictionnaire de musique», in JeanJacques Rousseau, Euvres complètes, 5. Écrits sur la musique, la langue et le théâtre. Paris, Gallimard ("Bibliothèque de la Pléiade») : CCLXIX-CCXVII.

\section{Guetti, Barbara H.}

1969 "The Double Voice of Nature :

Rousseau's Essai sur l'origine des langues", Modern Language Notes 84 (6) : 853-875.

Haddock, B. A.

1979 "Vico's "Discovery of the True Homer" : A Case-Study in Historical Reconstruction ", Journal of the History of Ideas 40 (4) : 583-602.

\section{Haugen, Kristine Louise}

1998 «Ossian and the Invention of Textual History ", Journal of the History of Ideas 59 (2) : 309-327. 
Hutton, C.A.

1927 "The Travels of "Palmyra" Wood in 1750-51 ", Journal of Hellenic Studies 47 (1) : 102-128.

Jousse, Marcel

1969 L'Anthropologie du geste.

Paris, Resma.

Kawada, Junzo

1998 La Voix. Étude d'ethno-linguistique comparative. Paris, Éd. de l'École

des hautes études en sciences sociales.

\section{Kintzler, Catherine}

1993 "Introduction", in J.-J. Rousseau, Essai sur l'origine des langues où il est parlé de la mélodie et de l'imitation musicale. Paris, Garnier-Flammarion : 5-46.

2006 [1991] Poétique de l'opéra français de Corneille à Rousseau. Paris, Minerve.

Kirk, Geoffrey S.

1999 [1965] Homer and the Epic.

Cambridge, Cambridge University Press.

Lévi-Strauss, Claude

1955 Tristes Tropiques. Paris, Plon.

\section{Lord, Albert Bates}

1960 The Singer of Tales. Cambridge, Harvard University Press.

1991 Epic Singers and Oral Tradition. Ithaca-London, Cornell University Press.

\section{Magnien, Victor}

1925 "Introduction ", in François Hélelin, abbé d'Aubignac, Conjectures académiques ou dissertation sur l'Iliade. Nouvelle édition corrigée, annotée, précédée d'une introduction par Victor Magnien. Paris, Librairie Hachette : I-XLVI.

Michelet, Jules

1827 "Discours sur le système et la vie de l'auteur [J. B. Vico] ", in Giambatista Vico, Principes de la philosophie de l'histoire. Traduits de la Scienza Nuova de J. B.Vico. Paris, J. Renouard : I-XLIX.
Minchin, Elizabeth

2001 Homer and the Resources of Memory. Some Applications of Cognitive Theory to the Iliad and the Odyssey. Oxford, Oxford University Press.

\section{Nagy, Gregory}

1996 Homeric Questions. Austin, University of Texas Press.

1999 "Les éditions alexandrines d'Homère au XVIII et au XIX siècle", in Françoise Létoublon \& Catherine Volpilhac-Auger, eds, Homère en France après la Querelle (17151900). Actes du colloque de Grenoble (23-25 octobre 1995), Université Stendhal-Grenoble 3. Paris, Honoré Champion : 41-49.

2000 [1996] La Poésie en acte. Homère et autres chants. Traduit de l'anglais par Jean Bouffartigue. Paris, Belin.

Nerval, Gérard de

1950 Voyage en Orient. Paris, Éditions Richelieu, 5 vol.

Norman, Larry F.

2005 "Homère entre anciens et modernes, 1670-1770», Lalies 25 : 59-110.

\section{Parry, Adam}

1956 "The Language of Achilles ", Transactions of the American Philological Association 87 : 1-7.

1966 "Have we Homer's Iliad? ", Yale Classical Studies 20 : 177-216.

Parry, Milman

1928 L'Épithète traditionnelle dans Homère. Paris, Les Belles Lettres.

1971 [1923] "A Comparative Study of Diction as one of the Elements of Style in Early Greek Epic Poetry ", in Adam Parry, ed., The Making of Homeric Verse.

The Collected Papers of Milman Parry.

Oxford, Clarendon Press : 421-435.

\section{Perrault, Charles}

1692 Parallèle des Anciens et des Modernes, 3. En ce qui regarde la poésie. Paris, Jean-Baptiste Coignard. 


\section{Perrin, Jean-François}

1999 "Comment Rousseau écrivait-il avec Homère? ", in Françoise Létoublon \& Catherine Volpilhac-Auger, eds, Homère en France après la Querelle (1715-1900). Actes du colloque de Grenoble (23-25 octobre 1995), Université Stendhal-Grenoble 3. Paris, Honoré Champion : 288-304.

\section{Porter, James}

2001 " "Bring me the Head of James

Macpherson" : The Execution of Ossian and the Wellsprings of Folkloristic Discourse ", The Journal of American Folklore

114 (454) : 396-435.

\section{Pot, Olivier}

1995 "Lettre sur la musique françoise " in Jean-Jacques Rousseau, Euvres complètes, 5. Écrits sur la musique, la langue et le théâtre. Paris, Gallimard ("Bibliothèque de la Pléiade ») : XCIX-CXXXV.

\section{Proust, Marcel}

1954 À la Recherche du temps perdu. Paris, Gallimard ("Bibliothèque de la Pléiade»), 3 vol.

Robinson, Philip E. J.

1984 Jean-Jacques Rousseau's Doctrine of the Arts. Bern, Peter Lang.

\section{Rossi, Luigi Enrico}

1999 «La méthode philologique de Jean Baptiste Gaspard d'Ansse de Villoison, et le Venetus Marcianus A ", in Françoise Létoublon \& Catherine Volpilhac-Auger, eds, Homère en France après la Querelle (1715-1900). Actes du colloque de Grenoble (23-25 octobre 1995), Université StendhalGrenoble 3. Paris, Honoré Champion : 51-61.

\section{Rousseau, Jean-Jacques}

1959 Euvres complètes, 1. Les Confessions, autres textes autobiographiques. Paris, Gallimard ("Bibliothèque de la Pléiade»).

1995 Euvres complètes, 5. Écrits sur la musique, la langue et le théâtre. Paris, Gallimard ("Bibliothèque de la Pléiade»).

\section{Simonsuuri, Kirsti}

1979 Homer's Original Genius. EighteenthCentury Notions of the Earl Greek Epic
University Press.

\section{Sosso, Paola}

1999 Jean-Jacques Rousseau. Imagination, illusions, chimères. Paris, Honoré Champion.

\section{Spencer, Terence J. B.}

1957 « Robert Wood and the Problem of Troy in the Eighteenth Century", Journal of the Warburg and Courtauld Institute $20(1-2)$ : 75-105.

Starobinski, Jean

1971 [1957] Jean-Jacques Rousseau.

La transparence et l'obstacle. Paris, Gallimard.

Tiersot, Julien

1920 Jean-Jacques Rousseau.

Paris, Félix Alcan.

\section{Tomlinson, Gary}

1999 «Vico's Songs : Detours at the

Origins of (Ethno) Musicology ",

The Musical Quaterly 83 (3) : 344-377.

\section{Touchefeu, Yves}

1995 "Rousseau et Homère ",

Dix-huitième siècle 27 : 179-190.

Verba, Cynthia

1989 «Jean-Jacques Rousseau : Radical and Traditional Views in His Dictionnaire de musique", The Journal of Musicology 7 (3) : 308-326.

Vico, Giambattista

1827 [1744] Principes de la philosophie de l'histoire. Traduits de la Scienza Nuova de J. B. Vico. Traduction de Jules Michelet. Paris, Renouard.

Viglieno, Laurence

2001 «La fête et l'esprit national selon Jean-Jacques Rousseau ", in Jean-Jacques Rousseau, politique et nation. Actes du 
II Colloque international de Montmorency. Paris, Honoré Champion : 831-845.

\section{Wade-Gery, Henry Theodore}

1952 The Poet of the Iliad. Cambridge, Cambridge University Press.

\section{Wolf, Friedrich August,}

1985 [1795] Prolegomena to Homer.

Traduit avec introduction et notes par
Anthony Grafton, Glenn W. Most \& James E. G. Zetzel. Princeton, Princeton University Press.

\section{Wood, Robert}

1973 [1775] An Essay on the Original

Genius and Writings of Homer.

With a Comparative View of the Ancient

and Present State of the Troade.

Washington, McGrath Publishing

Company.
Dominique Casajus, Quelques jours de la vie d'Homère. - La question de savoir comment l'Iliade et l'Odyssée ont été écrites s'est posée dès l'Antiquité. Le présent article parcourt quelques-unes des réponses qui lui ont été apportées à l'époque moderne. Pour l'abbé d'Aubignac, qui écrivait au milieu du XVII siècle, Homère n'avait jamais existé, et les ouvrages transmis sous son nom n'étaient qu'un amas de pièces auparavant dispersées. On ne cessa pourtant pas dans les siècles suivants d'écrire des "vies" d'Homère, ou du moins de brosser des portraits d'Homère au travail. Pour Giambattista Vico, Jean-Jacques Rousseau, Robert Wood, et plus tard Milman Parry et Albert Lord, Homère n'était pas un homme de plume mais un barde sans doute illettré, ce qui en faisait un poète supérieur à tous ceux qui lui avaient succédé : là où d'autres écrivaient, lui chantait. Cette valorisation nostalgique des cultures de la voix, beaucoup de spécialistes de la littérature orale la feraient leur encore aujourd'hui.
Dominique Casajus, A few Days in the Life of Homer. - How were the Iliad and Odyssey written is a question asked since Ancient times. Some of the answers provided during the modern period are presented. According to the abbey of Aubignac, writing in the mid- $17^{\text {th }}$ century, Homer never existed ; and the works passed down under his name are collections of previously disparate material. Nonetheless, the following centuries witnessed several "lives » of Homer or writings that tried to depict Homer at work. For Giambattista Vico, Jean-Jacques Rousseau, Robert Wood and, later on, Milman Parry and Albert Lord, Homer was not a writer but a bard, probably illiterate. This placed him as a poet far above all those who came afterwards: others wrote, but Homer sang. Attaching a nostalgic value to oral culture is something that many specialists of traditional literature would still do. 